VOL. 68 (2003) [141-153]

\title{
SOME FINITE SOLVABLE GROUPS WITH NON-TRIVIAL LATTICE ENDOMORPHISMS
}

\author{
S.E. STONEHEWER AND G. ZACHER
}

\begin{abstract}
The main purpose of this paper is to exhibit a doubly-infinite family of examples which are extensions of a $p$-group by a cyclic $p^{\prime}$-group, with the action satisfying some conditions of Zappa (1951), arising from his study of dual-standard (meetdistributive) subgroups. The examples show that Zappa's conditions do not bound the nilpotency class (or even the derived length) of the $p$-group. The key to this work is found in closely related conditions of Hartley (published here for the first time). The examples use some exceptional relationships between primes.
\end{abstract}

\section{INTRODUCTION}

In a group $G$ the map $X \mapsto X \cap D$ defines a subgroup-lattice endomorphism as soon as $D$ is a meet-distributive (dual-standard) subgroup of $G$, that is, $\langle X \cap D, Y \cap D\rangle$ $=\langle X, Y\rangle \cap D$ for all subgroups $X, Y$ of $G$. The dual-standard subgroups in finite groups have been characterised by Zappa [12] (see also [11, Theorem 11 in Section 7 of Chapter III]); he proves the following result.

THEOREM. Let $D$ be a dual-standard subgroup of a finite group $G$. Then $D$ is normal in $G$ and there are Hall subgroups $M, H, L$ of $G$ with $H$ nilpotent, such that $G$ is a split extension of $M \times L$ by $H, L \leqslant D \leqslant H L$ and $\pi(H L / D)=\pi(D / L)$. Also the Sylow subgroups of $H$ are cyclic or generalised quaternion.

Here $\pi(G)$ denotes the set of primes dividing the order of $G$. Further details are given in [10], where it is proved that the commutator subgroup $[H, L]$ is nilpotent. In this connection the action of a $p^{\prime}$-group $Q$ on a $p$-group $P$ ( $H$ and $L$ respectively in Zappa's Theorem) becomes relevant when there is a subgroup $Q_{1}$ of $Q$ such that

(Z1) for $X \leqslant P, Q_{1}$ normalises $X$ implies that $Q$ normalises $X$; and (Z2) $\quad C_{P}(Q)=C_{P}\left(Q_{1}\right)$.

Here $C_{P}(Q)$ is the centraliser of $Q$ in $P$. We refer to (Z1) and (Z2) by saying that the action of $Q$ on $P$ satisfies Zappa's conditions with respect to $Q_{1}$. The following properties are closely related:

(H1) $P$ has a $Q$-composition series

Received 13th January, 2003

The authors are grateful to CNR and MIUR for financial support during the preparation of this paper.

Copyright Clearance Centre, Inc. Serial-fee code: 0004-9727/03 \$A2.00+0.00. 


$$
1=P_{0} \triangleleft P_{1} \triangleleft P_{2} \cdots \triangleleft P_{r}=P,
$$

which is also a $Q_{1}$-composition series;

(H2) if $V_{i}$ and $V_{j}$ are composition factors of (1), then $V_{i}$ and $V_{j}$ are $Q$ isomorphic whenever they are $Q_{1}$-isomorphic; and

(H3) either $C_{P}\left(Q_{1}\right)=1$ or $C_{P}(Q) \neq 1$.

We refer to (H1), (H2) and (H3) by saying that the action of $Q$ on $P$ satisfies Hartley's conditions with respect to $Q_{1}$.

In this context it is natural to ask if the nilpotency class or derived length of $P$ is restricted by Zappa's conditions. In private communications in 1990, Brian Hartley showed that neither the class nor the derived length is bounded and, in order to do this, he introduced the conditions ( $\mathrm{H} 1),(\mathrm{H} 2)$ and (H3).

The present paper is divided in four sections. In the second we give Hartley's previously unpublished work, which is in two parts. Theorem 2.1 proves that Hartley's conditions imply Zappa's conditions when $Q$ is Abelian. Then examples are constructed to show that Zappa's conditions do not imply the existence of a bound on the derived length of $P$, again when $Q$ is Abelian. Section 3 deals with the relationship between Zappa's and Hartley's conditions, showing that in certain situations the former imply the latter. We also give more examples showing that (Z1) and (Z2) do not bound the nilpotency class of $P$. Finally in section 4 we give examples to show that Zappa's conditions do not imply Hartley's conditions in general.

We should point out that when $Q$ is cyclic or generalised quaternion and $Q_{1}$ is not equal to 1 or $Q$ and has order 2 if $Q$ is not cyclic, then Zappa shows that (Z1) and (Z2) are necessary and sufficient for $P Q_{1}$ to be a dual-standard subgroup of $P Q$.

All groups considered here are finite.

\section{HaRtLEy'S RESUlts}

We note first of all that if (H1) and (H2) hold for a given $Q$-composition series of $P$, then, by the Jordan-Hölder Theorem, they hold for every composition series of $P$. In particular we may always assume that (1) is a refinement of a central series of $P$.

Theorem 2.1. (Hartley) Let $P$ be a $p$-group acted on by an Abelian $p^{\prime}$-group $Q$ and let $Q_{1}$ be a subgroup of $Q$. Then

(i) with respect to $Q_{1},(\mathrm{H} 1)$ and (H2) imply (Z1).

Moreover

(ii) if in addition (H3) holds, then (Z2) also holds.

Proof: (i) Let $P$ have order $p^{n}$. We may assume that $n \geqslant 2$ and we proceed by induction on $n$. Let $X$ be a proper non-trivial subgroup of $P$ and suppose that $Q_{1}$ 
normalises $X$. Then we have to show that $Q$ normalises $X$. If $\Phi(P) \neq 1$, then, by induction, $X \Phi(P)$ is $Q$-invariant, and since

$$
X \leqslant X^{Q} \leqslant X \Phi(P) \neq P,
$$

again by induction it follows that $X$ is $Q$-invariant. Thus we may assume that $P$ is elementary Abelian.

Decompose $P$ under the action of $Q$ into its homogeneous components:

$$
P=W_{1} \oplus \cdots \oplus W_{r} .
$$

By (H1) and (H2), the $W_{i}$ here are also the homogeneous components for the action of $Q_{1}$. So there exists $i$ such that $X \cap W_{i} \neq 0$. If $r>1$, then, by induction, $X \cap W_{i}$ is $Q$-invariant. So factoring by $X \cap W_{i}$, we see, again by induction, that $X$ is $Q$-invariant. Therefore we may assume that $r=1$ and $X \leqslant P=W_{1}$.

Let $P=V_{1} \oplus \cdots \oplus V_{t}$ be a decomposition into $F_{p} Q$-modules. (Here $F_{p}$ is the field of $p$ elements.) For $1 \leqslant i, j \leqslant t, V_{i}$ and $V_{j}$ are $Q$-isomorphic and so they are $Q_{1}$ isomorphic. By $(\mathrm{H} 1), V_{i}$ and $V_{j}$ are irreducible $Q_{1}$-modules. Let $V_{i}$ have dimension $m$ over $F_{p}$. Since $Q$ is Abelian, it follows from [3, Proposition 8.2 on p. 154] that $P$ has $\left(p^{m t}-1\right) /\left(p^{m}-1\right)$ irreducible $F_{p} Q$ - as well as $F_{p} Q_{1}$-modules. But the first set is contained in the second, by (H1), hence the two sets coincide. Therefore $X$ is $Q$-invariant.

(ii) Let $C_{P}(Q)$ have order $p^{\beta}$. We may assume that $\beta \geqslant 1$. Also $\beta$ is the number of composition factors in a $Q$-composition series of $P$ on which $Q$ acts trivially. (This can be seen by choosing a $Q$-composition series of $P$ through $C_{P}(Q)$ and arguing by induction on the order of $P$. If $Q$ centralises a composition factor above $C_{P}(Q)$, then we may assume that it is $P / P_{r-1}$ and $P_{r-1}=\Phi(P)$. But then $Q$ centralises $P$.) Viewing such a series as a $Q_{1}$-composition series, it follows from (H2) that the number of trivial $Q_{1}$-composition factors cannot increase. Hence $C_{P}\left(Q_{1}\right)=C_{P}(Q)$.

Before we come to the construction of examples showing that there is no bound on the derived length of $P$ when Zappa's conditions are satisfied, we recall some known facts about faithful irreducible $Q$-modules $V$ over $F_{p}$ when $Q$ is a cyclic $q$-group and $q$ is a prime different from $p$. Let $Q=\langle\eta\rangle$ be a cyclic group of order $q^{e}$. Then $\operatorname{dim} V=\left|p \bmod q^{e}\right|=m$, say. If $\varepsilon$ is a primitive $q^{e}$-th root of 1 in $F_{p^{m}}$, then the map

$$
\eta^{i}: \nu \mapsto \nu \varepsilon^{i}
$$

defines a faithful irreducible $\langle\varepsilon\rangle$-module structure on the vector space $V_{\varepsilon}=F_{p}[\varepsilon]$ with basis $B=\left\{\varepsilon, \varepsilon^{p}, \ldots, \varepsilon^{p^{m-1}}\right\}$. If $\varepsilon^{\prime}$ is another primitive $q^{e}$-th root of 1 , then $V_{\varepsilon}$ and $V_{\varepsilon^{\prime}}$ are $Q$-isomorphic if and only if $\varepsilon^{\prime}$ belongs to $B$. The module $V$ is $Q$-isomorphic to some $V_{\varepsilon}$.

We need a combinatorial result concerning finite fields. 
Lemma 2.2. Let $e$ be a positive integer and let $p$ and $q$ be primes such that

$$
\left|p \bmod q^{e}\right|=m, \text { where } q^{e+1} \text { divides } p^{m}-1 .
$$

Then $F_{p^{m}}^{*}$ contains a subgroup $Q_{1}$ of order $q^{e}$. Let $\Gamma$ be the Galois group of $F_{p^{m}}$ over $F_{p}$. Suppose that $r$ is a positive integer such that

$$
(m+1)^{2(r-1)}<q^{e-1}(q-1) /\left(q^{e-1}+1\right) \quad \text { if } m \geqslant 2
$$

and

$$
2^{2(r-1)}<q-1 \text { if } m=1 .
$$

Then there exist generators $\lambda_{1}, \lambda_{2}, \ldots, \lambda_{r}$ of $Q_{1}$ such that the elements $\mu_{i_{1}} \mu_{i_{2}} \ldots \mu_{i_{s}}$, where $\left\{i_{1}, i_{2}, \ldots, i_{s}\right\}$ ranges over all subsets (including the empty set) of $\{1,2, \ldots, r\}$ and each $\mu_{j}$ ranges over the $\Gamma$-orbit of $\lambda_{j}$, are all distinct.

Proof: The Lemma is clear if $r=1$. So suppose that $r \geqslant 2$ and that we have found $\lambda_{1}, \lambda_{2}, \ldots, \lambda_{t}, 1 \leqslant t<r$. The number of elements $\mu_{i_{1}} \mu_{i_{2}} \ldots \mu_{i_{s}}$, with $\left\{i_{1}, i_{2}, \ldots, i_{s}\right\}$ a subset of of $\{1,2, \ldots, t\}$ and $\mu_{j}$ belonging to the $\Gamma$-orbit of $\lambda_{j}$, is at most

$$
\sum_{s=0}^{t}\left(\begin{array}{l}
t \\
s
\end{array}\right) m^{s}=(1+m)^{t} .
$$

If $\Lambda_{t}$ is this set of products, then we need to choose $\lambda_{t+1}=\lambda$ of order $q^{e}$ in $Q_{1}$, such that, for $\sigma$ in $\Gamma$, we have $\lambda^{\sigma} \notin \Lambda_{t} \Lambda_{t}^{-1}$, or equivalently $\lambda \notin \Lambda_{t} \Lambda_{t}^{-1}$ (since $\Lambda_{t} \Lambda_{t}^{-1}$ is $\Gamma$-invariant), and for $1 \neq \sigma \in \Gamma$,

$$
\lambda^{\sigma} \lambda^{-1}=\lambda^{p^{i}-1} \notin \Lambda_{t} \Lambda_{t}^{-1}
$$

$0 \leqslant i \leqslant m-1$. Recall that $q^{e}$ does not divide $p^{m-1}-1$. Thus raising to the power $p^{i}-1$ in $\langle\lambda\rangle$ is an endomorphism with kernel of order at most $q^{e-1}$. It follows that we must avoid at most $(m+1)^{2 t}$ values for $\lambda$ if $m=1$ and at most $(m+1)^{2 t}+q^{e-1}(m+1)^{2 t}$ values if $m \geqslant 2$. Thus if $m=1$, then the number to avoid is at most $2^{2(r-1)}$ and this is less than $q-1$ and therefore less that $q^{e}-q^{e-1}$. So we can choose a suitable $\lambda$. On the other hand, if $m \geqslant 2$, then we require

$$
(m+1)^{2(r-1)}+q^{e-1}(m+1)^{2(r-1)}<q^{e-1}(q-1),
$$

that is

$$
(m+1)^{2(r-1)}<q^{e-1}(q-1) /\left(q^{e-1}+1\right),
$$

which is the case. Again we can find $\lambda$. 
Now let $p, q$ be primes and let $e$ be a positive integer. Assume that $p$ has multiplicative order $m$ modulo $q^{e}$ and that $q^{n}$ is the highest power of $q$ dividing $p^{m}-1$, with $n>e$. In the finite field $F=F_{p^{m}}$, let $Q$ be the cyclic subgroup of $F^{*}$ of order $q^{f}$, where $e<f \leqslant n$; and let $\Gamma$ be the Galois group of $F$ over $F_{p}$. We have $\Gamma=\langle\sigma\rangle$, where $\sigma$ has order $m$. With this notation we can prove the following result.

THEOREM 2.3. (Hartley) Let $r$ be a positive integer such that

$$
(m+1)^{2(r-1)}<q^{e-1}(q-1) /\left(q^{e-1}+1\right) \quad \text { if } m \geqslant 2
$$

and

$$
2^{2(r-1)}<q-1 \quad \text { if } m=1 .
$$

Then there exists a $p$-group $P$ of nilpotency class $r$ and derived length $\left[\log _{2}(r+1)\right]$, acted upon faithfully by $Q$, such that Zappa's conditions are satisfied with respect to the subgroup $Q_{1}$ of order $q^{e}$.

Proof: By Lemma 2.2, we can find $r$ generators $\lambda_{1}, \lambda_{2}, \ldots, \lambda_{r}$ in $Q_{1}$. such that the elements $\mu_{i_{1}} \mu_{i_{2}} \ldots \mu_{i_{s}}$, where $\left\{i_{1}, i_{2}, \ldots, i_{s}\right\}$ ranges over all subsets of $\{1,2, \ldots, r\}$ and each $\mu_{j}$ ranges over the $\Gamma$-orbit of $\lambda_{j}$, are distinct.

Choose elements $\varepsilon_{1}, \varepsilon_{2}, \ldots, \varepsilon_{r}$ in $Q$ such that $\varepsilon_{i}^{q^{f-e}}=\lambda_{i}$ and let $V_{i}$ be the faithful irreducible $m$-dimensional $\left\langle\lambda_{i}\right\rangle$-module given by the action $\nu \mapsto \nu \lambda_{i}$, whose eigenvalues are the elements $\mu_{i}$ in the $\Gamma$-orbit of $\lambda_{i}$. Thus for $i_{1}<i_{2}<\cdots<i_{s}$ in $\{1,2, \ldots, r\}$, the eigenvalues of the $Q_{1}$-module

$$
V_{i_{1}} \otimes \cdots \otimes V_{i_{s}}
$$

where a generator of $Q_{1}$ acts. as multiplication on each $V_{i}$ by $\lambda_{i}$, are the elements $\mu_{i_{1}} \mu_{i_{2}} \ldots \mu_{i_{s}}$. These are pairwise distinct, by Lemma 2.2 . Consequently the module (2) splits into non-isomorphic irreducible $Q_{1}$-modules and, for different subsets of $\{1,2, \ldots, r\}$, they have no common constituents.

Let $P=H(V)$ be the Hartley $p$-group constructed from $V=\left\{V_{1}, V_{2}, \ldots, V_{r}\right\}$ (see [3, pp. 197-203]). Then $Q$ acts on $P$ as multiplication on each $V_{i}$ by $\varepsilon_{i}$. By [3, Theorem 12.8 on p. 202], the nilpotency class of $P$ is $r$ and the derived length is $\left[\log _{2}(r+1)\right]$. Under the action of $Q$, Hartley's conditions are satisfied with respect to $Q_{1}$. Therefore the result follows by Theorem 2.1 .

COROLLARY 2.4. Let $r$ be a positive integer and let $q$ be a prime greater than $2^{2(r-1)}$. Let $p$ be a prime such that $q^{2}$ divides $p-1$. Then there exists a $p$-group $P$ of nilpotency class $r$ and derived length $\left[\log _{2}(r+1)\right]$, acted on faithfully by a cyclic group $Q$ of order $q^{2}$, such that Zappa's conditions are satisfied with respect to $Q_{1}=Q^{q}$. 
Proof: Here $m=1$ and $e=1$. Note that by Dirichlet's Theorem, there are infinitely many primes $p$ satisfying our hypotheses.

Again with $e=1$, Theorem 2.3 also shows that there exists a 2-group $P$, of class $r$ and derived length $\left[\log _{2}(r+1)\right]$, acted upon faithfully by a cyclic group $Q$ of order $q^{2}$ such that Zappa's conditions hold with respect to $Q^{q}$, provided $q^{2}$ divides $2^{m}-1$ and $2(m+1)^{2(r-1)}<q-1$, where $m$ is the order of 2 modulo $q$ and may be a proper divisor of $q-1$.

Costantini has kindly pointed out to us that the existence of the prime $q$ is closely related to the so-called Wieferich primes, that is, primes $q$ such that $q^{2}$ divides $2^{q-1}-1$. The only Wieferich primes below $16.10^{12}$ are 1093 and 3511 ([1]). In our case we can take either of these values for $q$ when $r=1$.

\section{WHEN ZAPPA'S CONDITIONS IMPLY HARTLEY'S CONDITIONS}

The situation when $P$ is elementary Abelian is easy to deal with.

LEMma 3.1. Let $P$ be an elementary Abelian $p$-group on which a $p^{\prime}$-group $Q$ acts and let $Q_{1}$ be a subgroup of $Q$. Then with respect to $Q_{1},(\mathrm{Z} 1)$ implies (H1) and (H2).

Proof: Clearly (Z1) implies (H1). To show that (H2) holds, let

$$
P=V_{1} \oplus \cdots \oplus V_{t}
$$

be a decomposition of $P$ into irreducible $Q$-modules, therefore by (Z1) also irreducible $Q_{1}$-modules. Assume that for some $i \neq j, 1 \leqslant i, j \leqslant t$, there exists a $Q_{1}$-isomorphism $\gamma: V_{i} \rightarrow V_{j}$ and let $D_{\gamma}$ be the corresponding diagonal subgroup of $V_{i} \oplus V_{j}$. Since $D_{\gamma}$ is $Q_{1}$-invariant, it follows from (Z1) that it is also $Q$-invariant. Thus

$$
V_{i} \cong\left(V_{i} \oplus V_{j}\right) / D_{\gamma} \cong V_{j}
$$

that is $V_{i}$ and $V_{j}$ are $Q$-isomorphic. So (H2) holds.

We shall see in the next section that Zappa's conditions do not imply Hartley's conditions in general. However, other cases where (Z1) does imply (H1) and (H2) are contained in our next results.

A $p$-group $P$ is said to be powerful if $p$ is odd and $P / P^{p}$ is Abelian, or if $p=2$ and $P / P^{4}$ is Abelian. We recall from [2, Chapter 2] that when $P$ is powerful of exponent $p^{e}$, the Frattini series, defined by $P_{0}=P, P_{i+1}=\Phi\left(P_{i}\right)$ for $i \geqslant 0$, is a central series of $P$ of length $e$. Moreover, each term $P_{i}$ is also powerful and

$$
P_{i+1}=P_{i}^{p}=P^{p^{i+1}}
$$


Also $P$ has a basis $\left\{x_{1}, \ldots, x_{d}\right\}$ in the sense that each element of $P$ can be expressed uniquely in the form $x_{1}^{r_{1}} \cdots x_{d}^{r_{d}}$. Here $d$ is the minimal number of generators of $P$. (See [2, Exercise 9, Chapter 2].) By order considerations, the non-trivial elements of

$$
\left\{x_{1}^{p^{i}}, \ldots, x_{d}^{p^{i}}\right\}
$$

form a basis of $P_{i}$. Similarly $\left\{x_{1} P_{i}, \ldots, x_{d} P_{i}\right\}$ is a basis of $P / P_{i}$, for $1 \leqslant i \leqslant e$. For each $j$ we have

$$
\left\langle x_{j}\right\rangle \cap P_{i}=\left\langle x_{j}^{p^{i}}\right\rangle .
$$

Also $y=x_{1}^{r_{1}} \ldots x_{d}^{r_{d}}$ implies $\left|x_{i}^{r_{i}}\right| \leqslant|y|$, by [2, Lemma 2.4, (ii)].

THEOREM 3.2. Let $P$ be a powerful $p$-group of exponent $p^{e}$ and suppose that $\Omega\left(P / P_{i}\right)$ is Abelian for $1 \leqslant i \leqslant e$. Let $Q$ be a $p^{\prime}$-group acting on $P$ and let $Q_{1}$ be a proper, non-trivial subgroup of $Q$. If $(\mathrm{Z1})$ holds with respect to $Q_{1}$, then (H1) and (H2) also hold with respect to $Q_{1}$.

Proof: As always, (Z1) implies (H1). Thus suppose that (H2) does not hold and let $P$ be a counterexample of minimal exponent $p^{e}$. By Lemma 3.1, $e \geqslant 2$. Let

$$
1=X_{r} \triangleleft X_{r-1} \triangleleft \cdots \triangleleft X_{1} \triangleleft X_{0}=P
$$

be a $Q$-composition series of $P$ that refines the Frattini series. By choice of $P$, (H2) follows for $P_{i}$ and for $P / P_{i}$, for $1 \leqslant i \leqslant e-1$. Hence there are $i$ and $j$, with $P_{1} \leqslant X_{i}$ and $X_{j} \leqslant P_{e-1}$, such that $X_{i-1} / X_{i}$ and $X_{j} / X_{j+1}$ are $Q_{1}$-isomorphic, but not $Q$ isomorphic. Since $P / P_{1}$ and $P_{e-1}$ are completely reducible $Q$-modules, without loss of generality we may assume that $X_{i}=P_{1}$ and $j=r-1$.

Write $M=X_{i-1}$. Then $M / P_{1}$ is $Q$-irreducible. Let $y P_{1}$ be any non-trivial element of $M / P_{1}$. Let $\left\{x_{1}, \ldots, x_{d}\right\}$ be a basis of $P$ and let $y=x_{1}^{r_{1}} \ldots x_{d}^{r_{d}}$. Since $x_{i}^{r_{i}} \in P_{1}$ if $p$ divides $r_{i}$, we may (without changing $y P_{1}$ ) assume that

$$
y=x_{i_{1}}^{s_{1}} \cdots x_{i_{k}}^{s_{k}},
$$

where $p$ does not divide $s_{i}, 1 \leqslant i \leqslant k$. We distinguish two cases.

CASE 1. Suppose that $y$ has order $p^{s}$, where $s \geqslant 2$. The map $x \mapsto x^{p}$ induces a $Q$-epimorphism $\sigma: P / P_{1} \rightarrow P_{1} / P_{2}$, by [2, Lemma 2.4 (ii)]. Thus

$$
\left(y P_{1}\right)^{\sigma}=x_{i_{1}}^{p s_{1}} \cdots x_{i_{k}}^{p s_{k}} P_{2} \neq P_{2} .
$$

For, those elements $x_{i_{j}}^{p s_{j}}$, which are non-trivial, are (modulo $P_{2}$ ) part of a basis of $P_{1} / P_{2}$, as we observed above. They cannot all be trivial, otherwise $y$ has order $p$, which is not the case. Therefore (3) is true. Denoting the kernel of $\sigma$ by $K / P_{1}$, it follows that $K \cap M=P_{1}$. Thus $M / P_{1}$ is $Q$-isomorphic to a $Q$-composition factor lying between $P_{1}$ and $P_{2}$, which is a contradiction. 
CASE 2. Suppose that $y$ has order $p$. Here $y$ belongs to $\Omega(P)$, which is Abelian by hypothesis. Let $T=P_{1} \cap \Omega(P)$ and let $\Omega(P)=K \oplus T$ be a $Q$-decomposition. Now

$$
M / P_{1} \leqslant \Omega(P) P_{1} / P_{1} \cong \Omega(P) / T \cong K
$$

Hence $M / P_{1}$ is $Q$-isomorphic to some $Q$-invariant subgroup $B$ of $K$. Then $B$ and $P_{e-1}$ are $Q_{1}$-isomorphic, hence also $Q$-isomorphic, by Lemma 3.1. Thus $M / P_{1}$ and $P_{e-1}$ are $Q$-isomorphic, a contradiction.

Corollary 3.3. Let $P$ be a $p$-group acted on by a $p^{\prime}$-group $Q$ and let $Q_{1}$ be a proper, non-trivial subgroup of $Q$. If (Z1) holds with respect to $Q_{1}$, then (H1) and (H2) hold with respect to $Q_{1}$ whenever $P$ satisfies one of the following conditions:

(i) $P$ is a powerful group with a homogeneous basis $\left\{x_{1}, \ldots, x_{d}\right\}$, that is, $x_{i}$ and $x_{j}$ have the same order for all $i, j$;

(ii) $P$ is a modular group.

Proof: (i) By Theorem 3.2, it suffices to show that $\Omega\left(P / P_{i}\right)$ is Abelian for all $i$. Thus suppose that this is not the case and let $P$ be a counterexample of minimal exponent $p^{e}$. Clearly $e \geqslant 2$. For each $i<e, P / P_{i}$ also has a homogeneuos basis and exponent $p^{i}$. Hence $\Omega\left(P / P_{i}\right)$ is Abelian, by choice of $P$. Thus $\Omega(P)$ is not Abelian. Note that if $y$ has order $p$, then $y=x_{1}^{r_{1}} \cdots x_{d}^{r_{d}}$ and $x_{i}^{r_{i}}=1$ or $p$, as we observed above. Therefore $\Omega(P)=P_{e-1}=\left\langle x_{1}^{p^{e-1}}, \ldots, x_{d}^{p^{e-1}}\right\rangle$ is Abelian. a contradiction.

(ii) Now we suppose that $P$ is modular. Then $\Omega\left(P / P_{i}\right)$ is Abelian for all $i$, and it follows from Iwasawa's structure theorem (see [9, Theorem 2.3.1]) that if $P$ is not Hamiltonian, then $P$ is powerful. Thus Theorem 3.2 applies. On the other hand, if $P$ is Hamiltonian, then $P^{\prime}$ has order 2 and $P / P^{\prime}$ is elementary Abelian. Thus $P^{\prime}$ is centralised by $Q$ and so Lemma 3.1 applies.

REMARK. In [6] it is shown that a $p$-group $P$ is modular and non-Hamiltonian if and only if every subgroup of $P$ is powerful.

As applications of these results, we construct classes of $p$-groups $P$, acted upon by $p^{\prime}$-groups $Q$, in which Hartley's conditions are satisfied with respect to some proper, non-trivial subgroup $Q_{1}$ of $Q$.

EXAMPLE 3.4. Let $P$ be a special $p$-group (see [4, Kapitel 3, Section 13]), and let $Q$ be a $p^{\prime}$-group acting on $P$. Let $Q_{1}$ be a proper, non-trivial subgroup of $Q$. Suppose that $(\mathrm{Z} 1)$ and $(\mathrm{Z2})$ hold with respect to $Q_{1}$ and suppose also that $C_{P}(Q) \geqslant Z(P)$ if $P^{\prime} \neq 1$. Then, by Lemma 3.1, all of Hartley's conditions hold. When $p$ is odd, $P$ here is certainly powerful, but the hypothesis of Theorem 3.2 may not be satisfied. When $p=2$, then $P$ may not be powerful, but $C_{P}(Q) \geqslant Z(P)$ when $P$ is extraspecial.

Finally we show that the nilpotency class of $P$ is not restricted in these situations. 
Example 3.5. Let $p$ be a prime $\geqslant 5$ and let $A$ be an Abelian $p$-group of exponent $p^{m}, m \geqslant 2$. Consider the group $\Pi$ of power automorphisms of $A$. So $\Pi$ is cyclic, generated by $\alpha$, say, and

$$
\langle\alpha\rangle \cong C_{p^{m-1}} \times C_{p-1} .
$$

Let $P$ be the split extension of $A$ by $\langle\alpha\rangle_{p}$ and let $Q$ be the $p^{\prime}$-component of $\langle\alpha\rangle$. With $Q_{1}$ a proper, non-trivial subgroup of $Q$, one sees easily that when $Q$ acts on $P$, $(\mathrm{Z} 1)$ and $(\mathrm{Z} 2)$ are satisfied with respect to $Q_{1}$. So Theorem 3.2 applies.

\section{EXAMPLES WHERE ZAPPA'S CONDITIONS DO NOT IMPLY HARTLEY'S CONDITIONS}

Let $p$ be a prime and let $n$ and $c$ be integers $\geqslant 2$. Then let $P$ be a relatively free $p$-group of exponent $p$, rank $n$ and class $c$. So $P^{\prime}=\Phi(P)$ and $|P: \Phi(P)|=p^{n}$. Moreover, given a $p^{\prime}$-automorphism of $P / P^{\prime}$, it can be lifted to an automorphism $\eta$ of $P$ of the same order $([8])$. Let $Q=\langle\eta\rangle$ and suppose that $Q$ has order $q^{m}$, where $m>1$ and $q$ is a prime different from $p$. Assume that $V_{1}=P / P^{\prime}$ is a faithful irreducible $Q$-module, so that $F_{p^{n}}$ is a splitting field over $F_{p}$ of the polynomial $x^{q^{m}}-1$. For some primitive $q^{m}$ th root $\varepsilon$ of 1 in $F_{p^{n}}$, we have $V_{1} \cong V_{\varepsilon}$. Since the $p^{i}$ th powers of $\varepsilon$ ( $i$ going from 1 to $n-1$ ) form a basis of $V_{\varepsilon}$, it follows that there are $q^{m-1}(q-1) / n$ distinct faithful irreducible $Q$-modules. If we set $Q_{1}=\left\langle\eta^{q}\right\rangle$, then $V_{1}$ is also a faithful $Q_{1}$-module and it is irreducible if and only if $p$ has order $n$ modulo $q^{m-1}$.

Let $P=\gamma_{1}(P)>\gamma_{2}(P)>\gamma_{3}(P)>\cdots$ be the lower central series of $P$. Then $V_{i}=\gamma_{i}(P) / \gamma_{i+1}(P)$ is an $F_{p} Q$ - as well as an $F_{p} Q_{1}$-module. The map $\gamma$ from $V_{1} \times V_{i}$ to $V_{i+1}$ defined by

$$
\gamma:\left(x \gamma_{2}(P), y \gamma_{i+1}(P)\right) \mapsto[x, y] \gamma_{i+2}(P)
$$

is $\mathbb{Z}$-bilinear $\left(\left[\mathbf{5}\right.\right.$, p. 286]). Hence there exists an epimorphism $\gamma^{\prime}$ from $V_{1} \otimes V_{i}$ to $V_{i+1}$ mapping $\nu \otimes \nu_{i}$ to $\left(\nu, \nu_{i}\right)^{\gamma}$. We apply this construction in the next result.

PROPOSITION 4.1. Let $p$ be an odd prime and let $P$ be a 2-generator relatively free $p$-group of exponent $p$ and nilpotency class 3. Let $Q=\langle\eta\rangle$ be a group of automorphisms of $P$ of order $q^{2}$, where $q$ is a prime different from $p$. If $P / P^{\prime}$ is a faithful irreducible $Q$-module as well as an irreducible $\left\langle\eta^{q}\right\rangle$-module, then $P$ satisfies Hartley's conditions with respect to $Q_{1}=\left\langle\eta^{q}\right\rangle$. Also $q$ is odd.

Proof: We have $P^{\prime}=\Phi(P)>Z(P)>1$. Here, in the above notation, $n=2$, the dimension of $V_{2}$ is 1 and the dimension of $V_{3}$ is 2 . By assumption $\left|p \bmod q^{2}\right|$ $=|p \bmod q|=2$. It follows that $q^{2}$ divides $p^{2}-1$ and $q$ does not divide $p-1$. Hence $q^{2}$ divides $p+1$. Also $q$ must be odd.

Both $V_{1} \otimes V_{2}$ and $V_{3}$ have dimension 2. Hence $\gamma^{\prime}: V_{1} \otimes V_{2} \rightarrow V_{3}$ is an isomorphism and since $q$ does not divide $p-1, Q$ acts trivially on $V_{2}$. Therefore $V_{1}$ and $V_{3}$ are 
isomorphic $Q$-modules. Then (H1) and (H2) hold. Since $C_{P}(Q)$ and $C_{P}\left(Q_{1}\right)$ are equal (of order $p$ ), (H3) also holds.

Note that, by Theorem 2.1, Zappa's conditions also hold in the above situation.

In the remaining part of this paper we construct examples satisfying (Z1), (Z2), (H1) and (H3), but not (H2).

EXAMPLE 4.2. Let $p$ and $q$ be primes such that

$$
n=\left|p \bmod q^{2}\right|=|p \bmod q|>1 .
$$

Let $P$ be a relatively free $p$-group of exponent $p$, class 2 and rank $n$. With $V_{1}=P / P^{\prime}$ and $V_{2}=P^{\prime} / 1$, we know that there exists an epimorphism $\gamma$ from $V_{1} \otimes V_{1}$ to $V_{2}$, namely $\left(x P^{\prime} \otimes y P^{\prime}\right) \mapsto[x, y]$, whose kernel is $W=\left\langle\nu \otimes \nu \mid \nu \in V_{1}\right\rangle$. Thus

$$
V_{2} \cong\left(V_{1} \otimes V_{1}\right) / W=V_{1} \wedge V_{1} .
$$

By choice of $p$ and $q$, there exists an automorphism $\eta$ of $P$ of order $q^{2}$ such that, with $Q=\langle\eta\rangle$ and $Q_{1}=\left\langle\eta^{q}\right\rangle, V_{1}$ is a faithful irreducible $Q$ - as well as $Q_{1}$-module. Assume that $n=q-1$, which means that there exists only one faithful irreducible $Q_{1}$-module. Suppose also that there is no solution of the congruence

$$
p^{j} \equiv p^{i}+1 \bmod q^{2}
$$

for non-negative integers $i$ and $j$, with $i$ not divisible by $n$. For example $q=5, p=7$ will do.

Choose a $Q$-invariant subgroup $T$ of $P^{\prime}$ such that $V_{3}=P^{\prime} / T$ is a faithful irreducible $Q$-module. Let $V_{1} \cong V_{\varepsilon}$ for some primitive $q^{2}$ th root $\varepsilon$ of 1 in $F_{p^{n}}$. Then there exists $t$, with $0<t<n / 2$, such that with $s=p^{t}+1$ and $\lambda=\varepsilon^{s}, V_{\varepsilon} \cong V_{\lambda}$ $\left(\left[7\right.\right.$, p. 50]). Since the congruence relation (4) has no solution, $V_{1}$ and $V_{3}$ cannot be $Q$-isomorphic. It follows that the $Q$-group $P / T$ does not satisfy condition (H2) with respect to $Q_{1}$. Since the only $Q$-invariant subgroups in the interval $[P / T]$ are $P, P^{\prime}$ and $T$, and since $C_{P / T}(Q)=C_{P / T}\left(Q_{1}\right)=1$, Zappa's conditions are satisfied, while Hartley's condition (H2) fails to hold.

EXAmple 4.3. For our last example, take $p=67$ and let $P$ be a relatively free 3generator $p$-group of exponent $p$ and class 3. Let $Q=\langle\eta\rangle$ be a cyclic group of automorphisms of $P$ of order $q^{2}$, with $q=7$. Let $Q_{1}=\left\langle\eta^{7}\right\rangle$.

We have $\left|67 \bmod 7^{2}\right|=|67 \bmod 7|=3, P^{\prime}$ is an elementary Abelian $p$-group, and with $V_{1}=P / P^{\prime}, V_{2}=P^{\prime} / Z(P)$ and $V_{3}=Z(P) / 1, V_{1}$ and $V_{2}$ have dimension 3 , while $V_{3}$ has dimension 8 (over $F_{p}$ ). In $G L\left(V_{1}\right)$ we choose an element of order $q^{2}$, acting 
irreducibly, and lift it to an automorphism $\eta$ of $P$, still of order $q^{2}$. Then $V_{1}$ and $V_{2}$ are faithful irreducible $Q$ - as well as $Q_{1}$-modules, and they are not isomorphic (see [7, p. 52]). For a suitable primitive $q^{2}$ th root $\varepsilon$ of 1 in $F_{p^{3}}$, we have

$$
F_{p^{3}}=F_{p}[\varepsilon]=F_{p}\left[\varepsilon^{q}\right]
$$

and $V_{1}$ and $V_{\varepsilon}$ are $Q$-isomorphic, while with $\lambda=\varepsilon^{q}, V_{1}$ and $V_{\lambda}$ are $Q_{1}$-isomorphic. To get the minimal polynomial of $\varepsilon$ and $\lambda$ in $F_{p}[x]$, we factorise $x^{49}-1$ and $x^{7}-1$ into irreducible components. Thus

$$
\begin{aligned}
x^{49}-1= & (x-1)\left(x^{3}+13 x^{2}+x-1\right)\left(x^{3}+11 x^{2}+43 x-1\right)\left(x^{3}+40 x^{2}+33 x-1\right) \\
& \times\left(x^{3}+7 x^{2}+24 x-1\right)\left(x^{3}+32 x^{2}+62 x-1\right)\left(x^{3}+66 x^{2}+54 x-1\right) \\
& \times\left(x^{3}+43 x^{2}+60 x-1\right)\left(x^{3}+24 x^{2}+56 x-1\right)\left(x^{3}+5 x^{2}+35 x-1\right) \\
& \times\left(x^{3}+45 x^{2}+29 x-1\right)\left(x^{3}+34 x^{2}+27 x-1\right)\left(x^{3}+7 x^{2}+30 x-1\right) \\
& \times\left(x^{3}+37 x^{2}+60 x-1\right)\left(x^{3}+38 x^{2}+22 x-1\right)\left(x^{3}+12 x^{2}+11 x-1\right) . \\
& \times\left(x^{3}+56 x^{2}+55 x-1\right)
\end{aligned}
$$

and

$$
x^{7}-1=(x-1)\left(x^{3}+12 x^{2}+11 x-1\right)\left(x^{3}+56 x^{2}+55 x-1\right) .
$$

Let $f=x^{3}+13 x^{2}+x-1$. Then a root $\varepsilon$ of $f=f_{\varepsilon}$ in $F_{p^{3}}$ has order 49. Choose an automorphism $\eta$ of $P$ of order 49 and with the $F_{p} Q$-module $V_{1} Q$-isomorphic to $V_{\varepsilon}$. Then $\operatorname{trace}\left(\eta \mid V_{1}\right)=-13$.

If $x_{1}$ is a free generator of $P$, then $\left\{x_{1}, x_{2}\left(=x_{1}^{\eta}\right), x_{3}\left(=x_{2}^{\eta}\right)\right\}$ is a basis of $P$ and with $\nu_{i}=x_{i} P^{\prime}$ (in $V_{1}$ ), we get $\nu_{1}^{\eta}=\nu_{2}=\varepsilon \nu_{1}, \nu_{2}^{\eta}=\nu_{3}=\varepsilon \nu_{2}$ and

$$
\nu_{3}^{\eta}=\left(-13 \varepsilon^{2}-\varepsilon+1\right) \nu_{1}=\nu_{1}-\nu_{2}-13 \nu_{3}=x_{1} x_{2}^{-1} x_{3}^{-13} P^{\prime} .
$$

Let $w_{1}=\left[x_{1}, x_{2}\right] Z(P), w_{2}=\left[x_{2}, x_{3}\right] Z(P)$, and $w_{3}=\left[x_{3}, x_{1}\right] Z(P)$. Then $\left\{w_{1}, w_{2}, w_{3}\right\}$ is a basis of $V_{2}$ and we have $w_{1}^{\eta}=w_{2}, w_{2}^{\eta}=w_{2}+w_{3}$ and $w_{3}^{\eta}=w_{1}+13 w_{2}$. Therefore trace $\left(\eta \mid V_{2}\right)=1$, while trace $\left(\eta^{7} \mid V_{1}\right)=-12$ or -56 . (Using the Girard-Newton formulas, the exact value can be calculated from $f_{\varepsilon}$.) Now, as $F_{p} Q$-module, $V_{3}$ is a proper $Q$-epimorphic image of $V_{1} \otimes V_{2}$, since the dimension of $V_{1} \otimes V_{2}$ is 9 .

We have

$$
\operatorname{trace}\left(\eta \mid V_{1} \otimes V_{2}\right)=\operatorname{trace}\left(\eta \mid V_{1}\right) \times \operatorname{trace}\left(\eta \mid V_{2}\right)=-13,
$$

while

$$
\operatorname{trace}\left(\eta^{7} \mid V_{1} \otimes V_{2}\right)=\operatorname{trace}\left(\eta^{7} \mid V_{1}\right) \times \operatorname{trace}\left(\eta^{7} \mid V_{2}\right)=(-12)(-56)=2,
$$


since $V_{1}$ and $V_{2}$ are not $Q_{1}$-isomorphic. We conclude that the decomposition of $V_{3}$ into irreducible $Q_{1}$-modules is as follows:

$$
V_{3}=2 \times 1 \oplus U_{-12} \oplus U_{-56}
$$

(The indices here denote the trace of $\eta^{7}$ on these modules.) It follows that the only possibilities for the decomposition of the $Q$-module $V_{3}$ into a sum of irreducible submodules are

$$
2 \times 1 \oplus W_{-5} \oplus W_{-11}, \quad 2 \times 1 \oplus W_{-45} \oplus W_{-38}, \quad 2 \times 1 \oplus W_{-40} \oplus W_{-43},
$$

where the $W$ here are also faithful. From trace considerations, $W$ is not $Q$-isomorphic to $V_{1}$ or $V_{2}$. Thus one of the above 3 decompositions must be $V_{3}=2 \times 1 \oplus W \oplus U$, with $W \underset{\overline{Q_{1}}}{\cong} U_{-12}$ and $U \cong U_{-56}$. Hence, while $V_{1}$ is not $Q$-isomorphic to $W$ or $U$, $V_{1}$ is $Q_{1}$-isomorphic to $W$ or $U$. Therefore condition (H2) is not satisfied.

To see that Zappa's conditions hold, let $N=2 \times 1 \oplus U$ if $V_{1} \cong U_{-12}$ and let $N=2 \times 1 \oplus W$ if $V_{1} \cong U_{-56}$. Then $N$ is a normal $Q$-invariant subgroup of $P$. Also either $P^{\prime} / N \cong V_{2} \oplus W, P^{\prime} / N \cong V_{2} \oplus U_{-12}$ and $V_{2} \cong U_{-12}$ or $P^{\prime} / N \cong V_{2} \oplus U$, $P^{\prime} / N \underset{Q_{1}}{\cong} V_{2} \oplus U_{-56}$ and $V_{2} \cong U_{-56}$. In the interval $[P / N]$, every $Q$ - and $Q_{1}$-invariant subgroup different from $P$ and $P^{\prime}$ is properly contained in $\left[P^{\prime} / N\right]$, and this interval has exactly two $Q_{1}$-invariant subgroups, which are both $Q$-invariant. Hence Zappa's condition (Z1) is satisfied for $P / N$. Since $C_{P}(Q)=C_{P}\left(Q_{1}\right)$ is an elementary Abelian subgroup of order $p^{2}$ contained in $N$, condition (Z2) also holds for $P / N$. Thus finally we see that the $Q$-group $P / N$ satisfies Zappa's, but not Hartley's conditions, with respect to $Q_{1}$.

\section{REFERENCES}

[1] R. Crandall, C. Pomerance, Prime numbers, a computational perspective (Springer-Verlag, Berlin, 2001).

[2] J. Dixon, M. du Sautoy, A. Mann and D. Segal, Analytic pro-p-groups, London Mathematical Society Lecture Notes Series 157 (Cambridge University Press, Cambridge, 1991).

[3] K. Doerk and T.O. Hawkes, Finite soluble groups (de Gruyter, Berlin, New York, 1992).

[4] B. Huppert, Endliche Gruppen I (Springer-Verlag, Berlin, Heidelberg, New York, 1967).

[5] B. Huppert and N. Blackburn, Finite groups II (Springer-Verlag, Berlin, Heidelberg, New York, 1982).

[6] A. Lubotzky and A. Mann, 'Powerful p-groups I, finite groups', J. Algebra 105 (1987), 484-505.

[7] S. Mattarei, Retrieving information about a group from its character table, (Ph.D. Thesis) (University of Warwick, Warwick, 1992). 
[8] H. Neumann, Varieties of groups (Springer-Verlag, Berlin, Heidelberg, New York, 1967).

[9] R. Schmidt, Subgroup lattices of groups, de Gruyter Expositions in Mathematics 14 (de Gruyter, Berlin, New York, 1994).

[10] S.E. Stonehewer, G. Zacher, 'Dual-standard subgroups of finite and locally finite groups', Manuscripta Math. 70 (1991), 115-132.

[11] M. Suzuki, Structure of a group and the structure of its lattice of subgroups (Springer-Verlag, Berlin, Gottingen, Heidelberg, 1967).

[12] G. Zappa, 'Sulla condizione perché un emitrofismo inferiore tipico tra due gruppi sia un emotropismo', Giom. Mat. Battaglini 80 (1951), 80-101.

Mathematics Institute

University of Warwick

Coventry CV4 7AL

England

Stewart@stonehewer.freeserve.co.uk
Dipartimento di Matematica Pura ed Applicata Università di Padova

via Belzoni 7

35131 Padova

Italy

zacher@math.unipd.it 